\title{
Hypertrophic Lichen Planus-like Eruption Following Pembrolizumab
}

\author{
Stephanie Chapman, MD; Kurt Ashack, MD; Daniel C. Dapprich, MD
}

\section{PRACTICE POINTS}

- With an increased use of immunotherapy medications such as pembrolizumab for various cancers, it is important that dermatologists are aware of the wide range of adverse cutaneous reactions that can occur, including lichenoid reactions.

- Hypertrophic lichen planus should be considered in the differential diagnosis of patients with cutaneous lesions in addition to nail findings developing after starting programmed cell death protein 1 inhibitor therapy.

To the Editor:

Pembrolizumab, a humanized monoclonal antiprogrammed cell death protein 1 (PD-1) antibody, acts by blocking negative immune regulators such as PD- $1 .{ }^{1}$ Since its approval by the US Food and Drug Administration in 2014, the use of PD-1 inhibitors such as pembrolizumab has dramatically increased, and they are now the standard of care for cancers such as melanoma, lung cancer, and renal cell carcinoma. ${ }^{2,3}$ With increased use comes a better understanding of the cutaneous adverse effects that may occur. To date, almost $50 \%$ of patients treated with PD-1 inhibitors will develop an adverse cutaneous reaction. ${ }^{4}$ Thus far, cases of patients developing vitiligo, bullous pemphigoid, psoriasis, granulomatous skin reactions, severe cutaneous reactions (ie, toxic epidermal necrolysis), lupus erythematosus, and lichenoid reactions have been described. ${ }^{3,5,6}$ There are fewer than 30 documented cases of lichenoid reactions due to anti-PD-1 treatment described in the literature, increasing the importance of case reports to demonstrate a full range of cutaneous findings. ${ }^{3}$ We present a case of a reaction to pembrolizumab with an eruption of lichenoid papules predominantly involving the hands and feet as well as nail changes.

A 60-year-old man with ocular melanoma metastatic to the right lung, transverse colon, and right axillary lymph nodes presented with a chief concern of growing skin lesions present for 6 weeks on the hands and feet. The lesions were tender to the touch and occasionally drained a clear fluid. He also reported nail fragility. Of note, the patient was being treated for metastatic melanoma with pembrolizumab infusions every 3 weeks, which started 6 weeks prior to the onset of the eruption.

Physical examination demonstrated lichenoid papules on the dorsal and ventral aspects of the hands and feet (Figure 1), as well as longitudinal ridging on numerous fingernails and mild koilonychia. A punch biopsy revealed lichenoid interface dermatitis with irregular epidermal hyperplasia (Figure 2). A diagnosis of hypertrophic lichen planus-like drug eruption in response to pembrolizumab was made and clobetasol cream was prescribed.

At 1-month follow-up, the patient reported notable improvement with clobetasol, and he was transitioned to tacrolimus ointment $0.1 \%$. He continued to improve until a month later when he reported new lesions arising a week after a pembrolizumab infusion. He continued to use clobetasol cream for flares and tacrolimus ointment for maintenance.

Almost 3 months after the initial visit, the patient presented with inflammation around his right third fingernail

From the College of Human Medicine, Michigan State University, Grand Rapids. Dr. Chapman also is from the Department of Dermatology, Henry Ford Hospital, Detroit, Michigan. Drs. Dapprich and Ashack also are from the Dermatology Associates of West Michigan, Grand Rapids.

The authors report no conflict of interest.

Correspondence: Daniel C. Dapprich, MD, Dermatology Associates of West Michigan, 1740 E Paris Ave SE, Grand Rapids, MI 49546 (ddapprich@derm-associates.com).

doi:10.12788/cutis.0160 

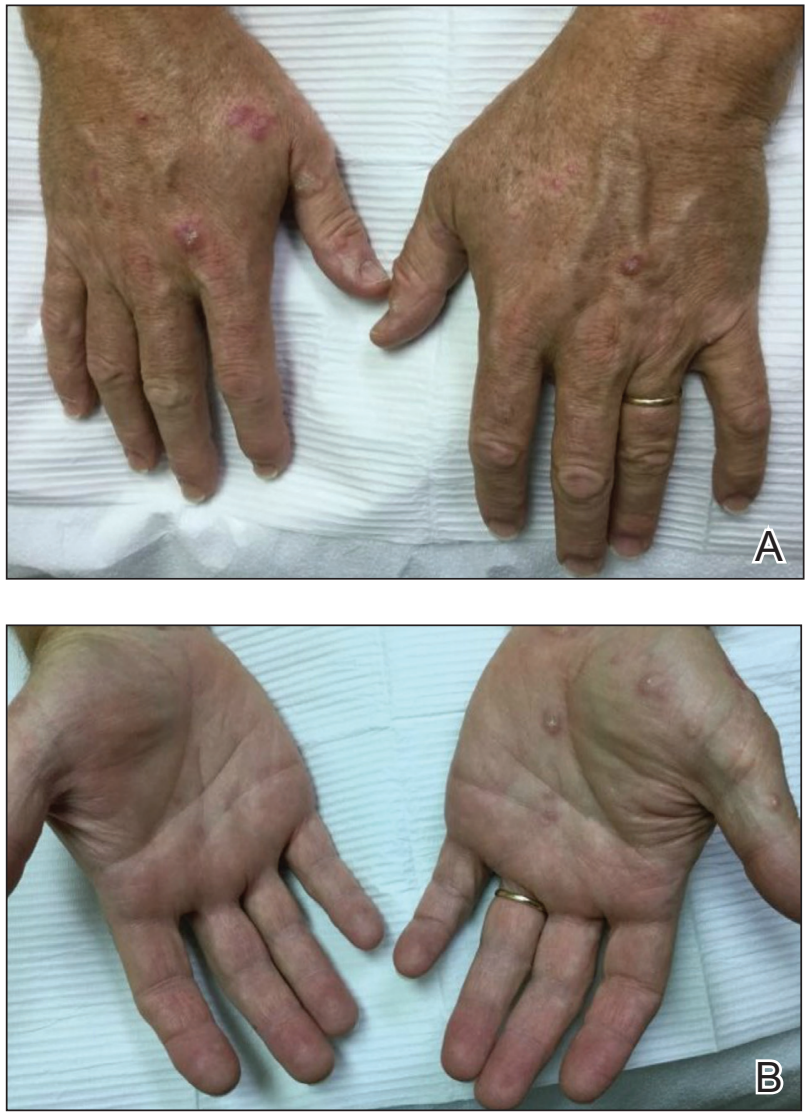

FIGURE 1. A and B, Lichenoid papules distributed on the ventral hands and dorsal hands, respectively.

of 1 week's duration, with more notable fragility than his other nails. No trauma was described, and the nail abnormality was attributed to pembrolizumab. Clobetasol cream and biotin $3 \mathrm{mg}$ daily resulted in improvement, and no other nails were affected in a similar way.

Programmed cell death protein 1 blockers are associated with a variety of adverse events including hypothyroidism, gastrointestinal abnormalities, fatigue, and skin disorders. ${ }^{7}$ In one study $(\mathrm{N}=83)$, cutaneous adverse drug events were found to occur in $42 \%$ (35/83) of patients following pembrolizumab therapy, with the most common cutaneous lesions being maculopapular eruptions (29\% [24/83]), pruritus (12\% [10/83]), and hypopigmentation $(8 \%[7 / 83]) .^{5}$

A total of 29 cases of lichenoid dermatitis following anti-PD-1 therapy have been described in the literature. ${ }^{3}$ Cases range from an eruption of photodistributed hyperkeratotic papules and plaques to hypertrophic vesiculobullous lesions. ${ }^{3,6}$ Suggested pathophysiology involves blocking the interaction of programmed death ligand 1 on keratinocytes with PD-1 on T cells. ${ }^{3}$ Management typically includes topical or systemic steroids. Cyclosporine

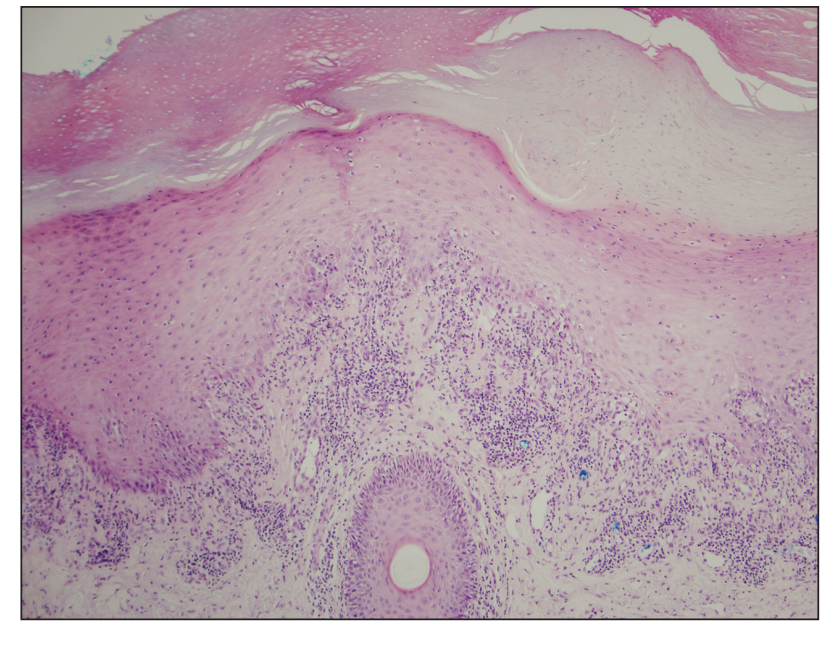

FIGURE 2. A punch biopsy showed lichenoid interface dermatitis with irregular epidermal hyperplasia (H\&E, original magnification $\times 100)$.

and acitretin also have been successful in a small number of patients. Most patients continue anti-PD-1 treatment with systemic therapy. ${ }^{3}$

Our patient represents a similar lichenoid eruption; however, the distribution on the dorsal and ventral aspects of the hands and feet as well as nail dystrophy make the presentation unique. Anticancer drugs that increase the T-cell immune response by altering the complex signaling among $\mathrm{T}$ cells, antigen-presenting cells, and tumor cells have been associated with cutaneous eruptions. Although the exact mechanism is still not fully understood, clinical suspicion of a pembrolizumab reaction should remain high on the differential in the setting of hyperkeratotic papules in association with anti-PD-1 therapy.

\section{REFERENCES}

1. Homet Moreno B, Ribas A. Anti-programmed cell death protein-1/ligand-1 therapy in different cancers. $\mathrm{Br} J$ Cancer. 2015;112:1421-1427.

2. Robert C, Ribas A, Wolchok JD, et al. Anti-programmed-deathreceptor-1 treatment with pembrolizumab in ipilimumab-refractory advanced melanoma: a randomised dose-comparison cohort of a phase 1 trial. Lancet. 2014;384:1109-1117.

3. Simonsen AB, Kaae J, Elleback E, et al. Cutaneous adverse reactions to anti-PD-1 treatment: a systematic review. J Am Acad Dermatol. 2020;83:1415-1424.

4. Hwang SJ, Carlos G, Wakade D, et al. Cutaneous adverse events (AEs) of anti-programmed cell death (PD)-1 therapy in patients with metastatic melanoma: a single-institution cohort. J Am Acad Dermatol. 2016;74:455-461.

5. Sanlorenzo M, Vujic I, Daud A, et al. Pembrolizumab cutaneous adverse events and their association with disease progression. JAMA Dermatol. 2015;151:1206-1212.

6. Joseph RW, Cappel M, Goedjen B, et al. Lichenoid dermatitis in three patients with metastatic melanoma treated with anti-PD-1 therapy. Cancer Immunol Res. 2015;3:18-22.

7. Hamid O, Robert C, Daud A, et al. Safety and tumor responses with lambrolizumab (anti-PD-1) in melanoma. N Engl J Med. 2013; 369:134-144. 